Commun. Korean Math. Soc. 23 (2008), No. 4, pp. 541-548

\title{
THE MINIMUM MODULUS OF A LINEAR MAP IN OPERATOR SPACES
}

\author{
Seung-Hyeok Kye
}

Reprinted from the Communications of the Korean Mathematical Society Vol. 23, No. 4, October 2008 


\title{
THE MINIMUM MODULUS OF A LINEAR MAP IN OPERATOR SPACES
}

\author{
SEUNG-HyEOK KYE
}

\begin{abstract}
For a completely bounded linear maps between operator spaces, we introduce numbers which measure the degree of injectivity and surjectivity. The number measuring the injectivity is an operator space analogue of the minimum modulus of a linear map in normed spaces.
\end{abstract}

\section{Introduction}

Subspaces and quotient spaces are key notions in Banach space theory. These notions can be expressed with isometries and quotient maps, which are special cases of injective and surjective linear maps, respectively. To be precise, we consider the following two numbers for a bounded linear map $\varphi: X \rightarrow Y$ between Banach spaces $X$ and $Y$ :

$$
\begin{aligned}
& \gamma(\varphi)=\inf \{\|\varphi(x)\|:\|x\|=1, x \in X\} \\
& \delta(\varphi)=\sup \left\{s \geq 0: \varphi\left(X_{1}\right) \supset Y_{s}\right\}
\end{aligned}
$$

respectively, where $X_{r}=\{x \in X:\|x\|<r\}$ for a positive number $r>0$.

Note that $\varphi$ is surjective if and only if $\delta(\varphi)>0$ by the open mapping theorem. If this is the case, then it is easy to see that the inequality

$$
\gamma(\varphi) \leq \delta(\varphi)
$$

holds. Although $\gamma(\varphi)>0$ implies the injectivity of $\varphi$, the converse does not hold in general. To see this, consider the set-theoretical inclusion map from $\ell^{1}(\mathbb{N})$ into $\ell^{\infty}(\mathbb{N})$. If $\varphi$ is injective, then the inequality

$$
\delta(\varphi) \leq \gamma(\varphi)
$$

also holds. If $\varphi$ is a contraction, then it is easy to see that $\varphi$ is an isometry if and only if $\gamma(\varphi)=1$, and $\varphi$ is a quotient map if and only if $\delta(\varphi)=1$. Therefore, the numbers $\gamma(\varphi)$ and $\delta(\varphi)$ measure the degree of injectivity and surjectivity of a linear map $\varphi$, respectively.

Received February 11, 2008.

2000 Mathematics Subject Classification. 47L25, 46B28.

Key words and phrases. minimum modulus, isometries, quotient maps, operator spaces. partially supported by KRF-2004-015-C00024. 
It is also easy to prove the identity

$$
\gamma\left(\varphi^{*}\right)=\delta(\varphi) \quad\left(\text { respectively } \delta\left(\varphi^{*}\right)=\gamma(\varphi)\right)
$$

whenever $\varphi$ is surjective (respectively injective) by using the bipolar theorem and Hahn-Banach extension theorem for Banach spaces. These equalities reflect the fact that $\varphi$ is an isometry (respectively a quotient map) if and only of $\varphi^{*}$ is a quotient map (respectively an isometry). The number $\gamma(\varphi)$ is called the minimum modulus of $\varphi$, and was introduced in [5] for the study of spectral theory of linear operators. A variation of this number

$$
\inf \{\|\varphi(x)\|: \operatorname{dist}(x, \operatorname{ker} \varphi)=1, x \in X\}
$$

was also introduced and studied in [6], [8], [1], [7].

After Ruan's thesis [11], the notion of operator spaces has been considered as a noncommutative quantum analogue of Banach spaces, and various aspects of operator spaces were studied extensively. We refer recent monographs [2], $[3],[10]$ for whole theory of operator spaces. One of the main topics in operator spaces is the notions of extension and lifting in various situations, as was in Banach space theory. These questions may be expressed in terms of tensor products as was explained in [9], where the numbers $\operatorname{proj}(V, W \subseteq Y)$ and $\operatorname{ex}(V, W \subseteq Y)$ were introduced to measure the possibilities of extension and lifting. These numbers are defined with the minimum modulus of suitable linear maps with respect to the tensor products. The purposes of this note are to define operator space analogues of the minimum modulus $\gamma_{\mathrm{op}}(\varphi)$ together with the number $\delta_{\mathrm{op}}(\varphi)$ for a completely bounded linear map $\varphi$ between operator spaces, and prove the operator space version of the identities in (2).

The author is grateful to Professor Woo Young Lee for bringing his attention to references [6], [8] for minimum modulus in Banach spaces.

\section{Preliminaries}

Every non-degenerate pairing $\langle, \quad\rangle$ of vector spaces $X$ and $Y$ determines the matrix pairing

$$
\langle,\rangle: M_{n}(X) \times M_{m}(Y) \rightarrow M_{m n}(\mathbb{C}), \quad m, n=1,2, \ldots
$$

by

$$
\left\langle\left[x_{i j}\right],\left[y_{k \ell}\right]\right\rangle=\left[\left\langle x_{i j}, y_{k \ell}\right\rangle\right]
$$

which is an $m n \times m n$ scalar matrix for $\left[x_{i j}\right] \in M_{n}(X)$ and $\left[y_{k \ell}\right] \in M_{m}(Y)$. More precisely, if

$$
\begin{aligned}
& x=\sum_{i, j=1}^{n} x_{i j} \otimes e_{i j} \in X \otimes M_{n}=M_{n}(X), \\
& y=\sum_{k, \ell=1}^{m} y_{k \ell} \otimes e_{k \ell} \in Y \otimes M_{m}=M_{m}(Y),
\end{aligned}
$$


then $\langle x, y\rangle$ is defined by

$$
\langle x, y\rangle=\sum_{i, j=1}^{n} \sum_{k, \ell=1}^{m}\left\langle x_{i j}, y_{k \ell}\right\rangle e_{i j} \otimes e_{k \ell} \in M_{n} \otimes M_{m},
$$

where $\left\{e_{i j}\right\}$ and $\left\{e_{k \ell}\right\}$ are usual matrix units for $M_{n}$ and $M_{m}$, respectively.

Given a graded set $\mathcal{D}=\left\{D_{n} \subset M_{n}(X): n \in \mathbb{N}\right\}$ of $X$, the absolute polar $\mathcal{D}^{\odot}$ is defined by the graded set $\left\{\mathcal{D}_{m}^{\odot} \subset M_{m}(Y): m \in \mathbb{N}\right\}$ of $Y$, where $\mathcal{D}_{m}^{\odot}$ is given by

$$
\mathcal{D}_{m}^{\odot}=\left\{\left[y_{k \ell}\right] \in M_{m}(Y):\left\|\left\langle\left[x_{i j}\right],\left[y_{k \ell}\right]\right\rangle\right\| \leq 1 \text { for each }\left[x_{i j}\right] \in D_{n}, n \in \mathbb{N}\right\}
$$

for each $m=1,2, \ldots$. We say that a graded set

$$
\mathcal{D}=\left\{D_{n} \subset M_{n}(X): n \in \mathbb{N}\right\}
$$

is absolutely matrix convex if

(i) $D_{m} \oplus D_{n} \subset D_{m+n}$

(ii) $a D_{m} b \subset D_{n}$ for each $a \in M_{n, m}$ and $b \in M_{m, n}$ with $\|a\| \leq 1$ and $\|b\| \leq 1$.

If $X$ is an operator space, then the graded set $\left\{M_{n}(X)_{1}: n \in \mathbb{N}\right\}$ of unit balls is a typical example of an absolute matrix convex set. It is also easy to see that if $\varphi: X \rightarrow Y$ is a linear map between operator spaces, then

$$
\left\{\varphi_{n}\left(M_{n}(X)_{1}\right) \subset M_{n}(Y): n \in \mathbb{N}\right\}
$$

is an absolutely matrix convex set, where $\varphi_{n}: M_{n}(X) \rightarrow M_{n}(Y)$ is defined by $\varphi_{n}\left(\left[x_{i j}\right]\right)=\left[\varphi\left(x_{i j}\right)\right]$. The absolute bipolar theorem [4], Proposition 4.1, says that if $\mathcal{D}$ is absolutely matrix convex set, then every set of $\mathcal{D}^{\odot \odot}$ is the weak closure of the corresponding set of $\mathcal{D}$, whenever $X$ and $Y$ are locally convex spaces with a non-degenerated pairing.

If $\mathcal{D}$ is an absolutely convex set, then the definition of $\mathcal{D}^{\odot}$ may be simplified by

$$
\mathcal{D}_{m}^{\odot}=\left\{\left[y_{k \ell}\right] \in M_{m}(Y):\left\|\left\langle\left[x_{i j}\right],\left[y_{k \ell}\right]\right\rangle\right\| \leq 1 \text { for each }\left[x_{i j}\right] \in \mathcal{D}_{m}\right\}
$$

This is already implicit in [4], Lemma 5.2, in terms of "gauges". We provide a simple proof with a similar argument as in there.

Let $x \in M_{n}(X)$ and $y \in M_{m}(Y)$ be given as in (3). If $a=\left[a_{s i}\right] \in M_{m, n}(\mathbb{C})$ and $b=\left[b_{j t}\right] \in M_{n, m}(\mathbb{C})$, then it is straightforward to see the following:

$$
a e_{i j} b=\sum_{s, t=1}^{m} a_{s i} b_{j t} e_{s t} \in M_{m}(\mathbb{C}), \quad i, j=1,2, \ldots, n .
$$


Since $a x b=\sum_{s, t=1}^{m}\left(\sum_{i, j=1}^{m} a_{s i} x_{i j} b_{j t}\right) \otimes e_{s t} \in M_{m}(X)$, we have

$$
\begin{aligned}
\langle a x b, y\rangle & =\sum_{s, t=1}^{m} \sum_{k, \ell=1}^{m}\left\langle\sum_{i, j=1}^{n} a_{s i} x_{i j} b_{j t}, y_{k \ell}\right\rangle e_{s t} \otimes e_{k \ell} \\
& =\sum_{i, j=1}^{n} \sum_{k, \ell=1}^{m}\left\langle x_{i j}, y_{k \ell}\right\rangle\left(a e_{i j} b\right) \otimes e_{k \ell} \in M_{m} \otimes M_{m}
\end{aligned}
$$

by (5). In order to prove (4), we assume that $y \in M_{m}(Y)$ satisfies

$$
\|\langle x, y\rangle\| \leq 1 \quad \text { for each } x \in \mathcal{D}_{m},
$$

and show the following:

$$
\|\langle x, y\rangle\| \leq 1 \quad \text { for each } x \in \mathcal{D}_{n} \text { and } n=1,2, \ldots
$$

If $n \leq m$, then there is nothing to prove, since $\mathcal{D}_{n} \subset \mathcal{D}_{m}$. Assume that $n>m$. If $\xi$ and $\eta$ are unit vectors in $\mathbb{C}^{m n}=\mathbb{C}^{n} \otimes \mathbb{C}^{m}$, then by [4], Lemma 5.1, there are isometries $a$ and $b$ from $\mathbb{C}^{m}$ into $\mathbb{C}^{n}$ and unit vectors $\bar{\xi}$ and $\bar{\eta}$ in $\mathbb{C}^{m} \otimes \mathbb{C}^{m}$ such that

$$
\xi=(a \otimes \mathrm{id})(\bar{\xi}), \quad \eta=(b \otimes \mathrm{id})(\bar{\eta}) .
$$

Therefore, we have

$$
\begin{aligned}
\langle\langle x, y\rangle \xi, \eta\rangle & =\sum_{i, j=1}^{n} \sum_{k, \ell=1}^{m}\left\langle x_{i j}, y_{k \ell}\right\rangle\left\langle\left(e_{i j} \otimes e_{k \ell}\right)(a \otimes \mathrm{id})(\bar{\xi}),(b \otimes \mathrm{id})(\bar{\eta})\right\rangle \\
& =\sum_{i, j=1}^{n} \sum_{k, \ell=1}^{m}\left\langle x_{i j}, y_{k \ell}\right\rangle\left\langle\left[\left(b^{*} e_{i j} a\right) \otimes e_{k \ell}\right] \bar{\xi}, \bar{\eta}\right\rangle \\
& =\left\langle\left\langle b^{*} x a, y\right\rangle \bar{\xi}, \bar{\eta}\right\rangle
\end{aligned}
$$

by (6), and the relation ( 7$)$ follows since $b^{*} x a \in \mathcal{D}_{m}$ by the absolute convexity of $\mathcal{D}$.

We need one more fact which is a simple application of the open mapping theorem: If $\varphi: X \rightarrow Y$ is a surjective bounded linear map, then we have

$$
Y_{s} \subset \overline{\varphi\left(X_{1}\right)} \Longrightarrow Y_{s} \subset \varphi\left(X_{1}\right) \text {. }
$$

First, we show that $r<s \Longrightarrow \overline{\varphi\left(X_{r}\right)} \subset \varphi\left(X_{s}\right)$. Assume that $r<s$. For $\varphi(x) \in$ $\overline{\varphi\left(X_{r}\right)}$, take an open ball $N$ of $x$ with radius $s-r$. Then $\varphi(N) \cap \varphi\left(X_{r}\right) \neq \emptyset$ since $\varphi(N)$ is an open set. Take $x_{1} \in N$ and $x_{2} \in X_{r}$ such that $\varphi\left(x_{1}\right)=\varphi\left(x_{2}\right)$. Then we have $\varphi(x)=\varphi\left(x-x_{1}+x_{2}\right)$ and

$$
\left\|x-x_{1}+x_{2}\right\| \leq\left\|x-x_{1}\right\|+\left\|x_{2}\right\|<(s-r)+r=s,
$$

and so $\varphi(x) \in \varphi\left(X_{s}\right)$. Now, if $Y_{s} \subset \overline{\varphi\left(X_{1}\right)}$, then $Y_{a s} \subset \overline{\varphi\left(X_{a}\right)} \subset \varphi\left(X_{1}\right)$ for any $a \in(0,1)$. Therefore, we have $Y_{s} \subset \varphi\left(X_{1}\right)$, and this proves (8).

Finally, we note that

$$
s \leq \delta(\varphi) \Longleftrightarrow \varphi\left(X_{1}\right) \supset Y_{s}
$$


for a positive number $s>0$. On the other hand, we also have

$$
\begin{aligned}
s \leq \gamma(\varphi) & \Longleftrightarrow\|x\|=1 \text { implies }\|\varphi(x)\| \geq s \\
& \Longleftrightarrow\|\varphi(x)\|<s \text { implies }\|x\|<1 \\
& \Longleftrightarrow\|\varphi(x)\| \leq s \text { implies }\|x\| \leq 1
\end{aligned}
$$

\section{Main result}

Note that the following theorem with $n=1$ gives the identities in (2), because every Banach space may be endowed with an operator space structure. We also note that if $\varphi_{n}$ is bounded, then the map $\left(\varphi^{*}\right)_{n}: M_{n}\left(Y^{*}\right) \rightarrow M_{n}\left(X^{*}\right)$ is also bounded.

Theorem 3.1. Let $\varphi: X \rightarrow Y$ be a bounded linear map between operator spaces $X$ and $Y$, with the dual map $\varphi^{*}: Y^{*} \rightarrow X^{*}$ between the operator duals $Y^{*}$ and $X^{*}$. Fix a natural number $n=1,2, \ldots$ and assume that the map $\varphi_{n}$ is bounded. Then we have the following:

(i) We have $\delta\left(\varphi_{n}\right) \leq \gamma\left(\left(\varphi^{*}\right)_{n}\right)$ : The equality $\delta\left(\varphi_{n}\right)=\gamma\left(\left(\varphi^{*}\right)_{n}\right)$ holds whenever $\varphi$ is surjective.

(ii) We have $\gamma\left(\varphi_{n}\right) \geq \delta\left(\left(\varphi^{*}\right)_{n}\right)$ : The equality $\gamma\left(\varphi_{n}\right)=\delta\left(\left(\varphi^{*}\right)_{n}\right)$ holds whenever $\varphi$ is injective.

Proof. For the first inequality, it suffices to show that $s \leq \delta\left(\varphi_{n}\right)$ implies $s \leq$ $\gamma\left(\left(\varphi^{*}\right)_{n}\right)$, or equivalently, the inclusion $\varphi_{n}\left(M_{n}(X)_{1}\right) \supset M_{n}(Y)_{s}$ implies the following relation

$$
\left\|\left[g_{i j}\right]\right\|_{M_{n}\left(Y^{*}\right)}=1 \Longrightarrow\left\|\left(\varphi^{*}\right)_{n}\left(\left[g_{i j}\right]\right)\right\|_{M_{n}\left(X^{*}\right)} \geq s
$$

by (9) and (10). Assume $\varphi_{n}\left(M_{n}(X)_{1}\right) \supset M_{n}(Y)_{s}$ and $\left\|\left[g_{i j}\right]\right\|_{M_{n}\left(Y^{*}\right)}=1$. Then we have

$$
\begin{aligned}
\left\|\left(\varphi^{*}\right)_{n}\left(\left[g_{i j}\right]\right)\right\|_{M_{n}\left(X^{*}\right)} & =\sup \left\{\left\|\left\langle\left[x_{k \ell}\right],\left[\varphi^{*}\left(g_{i j}\right)\right]\right\rangle\right\|:\left[x_{k \ell}\right] \in M_{n}(X)_{1}\right\} \\
& =\sup \left\{\left\|\left\langle\left[\varphi\left(x_{k \ell}\right)\right],\left[g_{i j}\right]\right\rangle\right\|:\left[x_{k \ell}\right] \in M_{n}(X)_{1}\right\} \\
& \geq \sup \left\{\left\|\left\langle\left[y_{k \ell}\right],\left[g_{i j}\right]\right\rangle\right\|:\left[y_{k \ell}\right] \in M_{n}(Y)_{s}\right\} \\
& =s \cdot \sup \left\{\left\|\left\langle\left[y_{k \ell}\right],\left[g_{i j}\right]\right\rangle\right\|:\left[y_{k \ell}\right] \in M_{n}(Y)_{1}\right\} \\
& =s \cdot\left\|\left[g_{i j}\right]\right\|_{M_{n}\left(Y^{*}\right)}=s
\end{aligned}
$$

and this proves $\delta\left(\varphi_{n}\right) \leq \gamma\left(\left(\varphi^{*}\right)_{n}\right)$.

Now, we assume that $\varphi$ is surjective and proceed to show

$$
\gamma\left(\left(\varphi^{*}\right)_{n}\right) \leq \delta\left(\varphi_{n}\right)
$$

To do this, we assume that $0<s \leq \gamma\left(\left(\varphi^{*}\right)_{n}\right)$ and show $s \leq \delta\left(\varphi_{n}\right)$. (If $\gamma\left(\left(\varphi^{*}\right)_{n}\right)=0$, then there is nothing to prove.) Note that $s \leq \gamma\left(\left(\varphi^{*}\right)_{n}\right)$ if and only if

$$
\left\|\left[\varphi^{*}\left(g_{k \ell}\right)\right]\right\|_{M_{n}\left(X^{*}\right)} \leq 1 \Longrightarrow\left\|\left[g_{k \ell}\right]\right\|_{M_{n}\left(Y^{*}\right)} \leq \frac{1}{s}
$$


by (10). Under this assumption, we show the relation

$$
M_{n}(Y)_{s} \subset\left[\left\{\varphi_{m}\left(M_{m}(X)_{1}\right): m \in \mathbb{N}\right\}^{\odot}\right]_{n}^{\odot} .
$$

To do this, we assume that $\left[y_{i j}\right] \in M_{n}(Y)_{s}$. If

$$
\left[g_{k \ell}\right] \in\left\{\varphi_{m}\left(M_{m}(X)_{1}\right): m \in \mathbb{N}\right\}_{n}^{\odot},
$$

then we have

$$
\begin{aligned}
\left\|\left[\varphi^{*}\left(g_{k \ell}\right)\right]\right\|_{M_{n}\left(X^{*}\right)} & =\sup \left\{\left\|\left\langle\left[x_{i j}\right],\left[\varphi^{*}\left(g_{k \ell}\right)\right]\right\rangle\right\|:\left[x_{i j}\right] \in M_{n}(X)_{1}\right\} \\
& =\sup \left\{\left\|\left\langle\left[\varphi\left(x_{i j}\right)\right],\left[g_{k \ell}\right]\right\rangle\right\|:\left[x_{i j}\right] \in M_{n}(X)_{1}\right\} \\
& \leq 1
\end{aligned}
$$

and so $\left\|\left[g_{k \ell}\right]\right\|_{M_{n}\left(Y^{*}\right)} \leq \frac{1}{s}$ by $(11)$. Since $\left[y_{i j}\right] \in M_{n}(Y)_{s}$, we have

$$
\left\|\left\langle\left[y_{i j}\right],\left[g_{k \ell}\right]\right\rangle\right\| \leq 1,
$$

and this proves the relation (12) by (4). By the absolute bipolar theorem, we have

$$
M_{n}(Y)_{s} \subset \overline{\varphi_{n}\left(M_{n}(X)_{1}\right)}, \quad n=1,2, \ldots
$$

Since $\varphi_{n}$ is surjective, we see that

$$
M_{n}(Y)_{s} \subset \varphi_{n}\left(M_{n}(X)_{1}\right), \quad n=1,2, \ldots
$$

by (8), as was desired.

To prove the inequality in (ii), we assume that

$$
\left(\varphi^{*}\right)_{n}\left(M_{n}\left(Y^{*}\right)_{1}\right) \supset M_{n}\left(X^{*}\right)_{s}
$$

and $\left\|\left[x_{i j}\right]\right\|_{M_{n}(X)}=1$. It suffices to show that $\left\|\varphi_{n}\left(\left[x_{i j}\right]\right)\right\|_{M_{n}(Y)} \geq s$, as in the proof of the first inequality of (i). Recall that $\left\|\left[y_{i j}\right]\right\|_{M_{n}(Y)}=\left\|\left[y_{i j}\right]\right\|_{M_{n}\left(Y^{* *}\right)}$, since the inclusion $Y \hookrightarrow Y^{* *}$ is a complete isometry. Therefore, we have

$$
\begin{aligned}
\left\|\varphi_{n}\left(\left[x_{i j}\right]\right)\right\|_{M_{n}(Y)} & =\sup \left\{\left\|\left\langle\left[g_{k \ell}\right],\left[\varphi\left(x_{i j}\right)\right]\right\rangle\right\|:\left[g_{k \ell}\right] \in M_{n}\left(Y^{*}\right)_{1}\right\} \\
& =\sup \left\{\left\|\left\langle\left[\varphi^{*}\left(g_{k \ell}\right)\right],\left[x_{i j}\right]\right\rangle\right\|:\left[g_{k \ell}\right] \in M_{n}\left(Y^{*}\right)_{1}\right\} \\
& \geq \sup \left\{\left\|\left\langle\left[f_{k \ell}\right],\left[x_{i j}\right]\right\rangle\right\|:\left[f_{k \ell}\right] \in M_{n}\left(X^{*}\right)_{s},\right\} \\
& =s \cdot \sup \left\{\left\|\left\langle\left[f_{k \ell}\right],\left[x_{i j}\right]\right\rangle\right\|:\left[f_{k \ell}\right] \in M_{n}\left(X^{*}\right)_{1}\right\} \\
& =s \cdot\left\|\left[x_{i j}\right]\right\|_{M_{n}(X)}=s,
\end{aligned}
$$

as was desired.

It remains to show the inequality $\gamma\left(\varphi_{n}\right) \leq \delta\left(\left(\varphi^{*}\right)_{n}\right)$, under the assumption that $\varphi$ is injective. We may assume that $\gamma\left(\varphi_{n}\right)>0$. We proceed to show

$$
0<s \leq \gamma\left(\varphi_{n}\right) \Longrightarrow s \leq \delta\left(\left(\varphi^{*}\right)_{n}\right) \text {. }
$$

If $\left[f_{i j}\right] \in M_{n}\left(X^{*}\right)_{s}$, then the map $f: X \rightarrow M_{n}$ given by $f(x)=\left[\left\langle x, f_{i j}\right\rangle\right]$ is completely bounded with $\|f\|_{\mathrm{cb}} \leq s$. Now, the assumption $s \leq \gamma\left(\varphi_{n}\right)$ shows that

(13) $\left\|\left[\varphi\left(x_{i j}\right)\right]\right\|_{M_{n}(Y)} \leq 1 \Longrightarrow\left\|\left[x_{i j}\right]\right\|_{M_{n}(X)} \leq \frac{1}{s} \Longrightarrow\left\|\left[f\left(x_{i j}\right)\right]\right\|_{M_{n}\left(M_{n}\right)} \leq 1$. 
If we regard $\varphi(X)$ as an operator subspace of $Y$, we have the map

$$
f_{1}: \varphi(X) \rightarrow M_{n}
$$

such that $f=f_{1} \circ \varphi$, and the relation (13) says that $\left\|f_{1}\right\|_{\mathrm{cb}}=\left\|\left(f_{1}\right)_{n}\right\| \leq 1$. By the Arveson-Wittstock Hahn-Banach theorem, there exists a completely contractive map $g: Y \rightarrow M_{n}$ which extends $f_{1}$. If $g=\left[g_{i j}\right] \in M_{n}\left(Y^{*}\right)$, then we see that $\left(\varphi^{*}\right)_{n}\left(\left[g_{i j}\right]\right)=\left[f_{i j}\right]$. Therefore, we have shown

$$
M_{n}\left(X^{*}\right)_{s} \subset\left(\varphi^{*}\right)_{n}\left(\overline{M_{n}\left(Y^{*}\right)_{1}}\right),
$$

which implies

$$
M_{n}\left(X^{*}\right)_{s} \subset\left(\varphi^{*}\right)_{n}\left(M_{n}\left(Y^{*}\right)_{1}\right)
$$

by (8), because our argument shows that $\left(\varphi^{*}\right)_{n}$ is surjective. Therefore, we have $s \leq \delta\left(\left(\varphi^{*}\right)_{n}\right)$, and this completes the proof.

It is easy to see that the sequences $\left\{\gamma\left(\varphi_{n}\right)\right\}$ and $\left\{\delta\left(\varphi_{n}\right)\right\}$ are decreasing. To see this, let $n<m$. We first show that $\gamma\left(\varphi_{m}\right) \leq \gamma\left(\varphi_{n}\right)$, or equivalently, $s \leq \gamma\left(\varphi_{m}\right)$ implies $s \leq \gamma\left(\varphi_{n}\right)$. Assume that $s \leq \gamma\left(\varphi_{m}\right)$, that is, $\left\|\left[a_{i j}\right]\right\|_{M_{m}(X)}=1$ implies $\left\|\left[\varphi\left(x_{i j}\right)\right]\right\|_{M_{m}(Y)} \geq s$ by $(10)$. If $\left\|\left[x_{k \ell}\right]\right\|_{M_{n}(X)}=1$, then $\left\|\left[\begin{array}{cc}x_{k \ell} & 0 \\ 0 & 0\end{array}\right]\right\|_{M_{m}(X)}=1$, which implies that

$$
\left\|\left[\varphi\left(x_{k \ell}\right)\right]\right\|_{M_{n}(Y)}=\left\|\left[\begin{array}{cc}
\varphi\left(x_{k \ell}\right) & 0 \\
0 & 0
\end{array}\right]\right\|_{M_{m}(Y)} \geq s .
$$

Therefore, we have $\gamma\left(\varphi_{n}\right) \geq s$ by (10). For the inequality $\delta\left(\varphi_{m}\right) \leq \delta\left(\varphi_{n}\right)$, we assume that $\varphi_{m}\left(M_{m}(X)_{1}\right) \supset M_{m}(Y)_{s}$ and show $\varphi_{n}\left(M_{n}(X)_{1}\right) \supset M_{n}(Y)_{s}$. If $y \in M_{n}(Y)_{s}$, then $y^{\prime}=\left[\begin{array}{ll}y & 0 \\ 0 & 0\end{array}\right]$ lies in $M_{m}(Y)_{s}$, and so there is $x^{\prime} \in M_{m}(X)_{1}$ such that $\varphi_{m}\left(x^{\prime}\right)=y^{\prime}$. If we take the $m \times m$ submatrix $x$ of $x^{\prime}$ from the left upper corner of $x^{\prime}$, then $x \in M_{n}(X)_{1}$, and so $y=\varphi_{n}(x) \in \varphi_{n}\left(M_{n}(X)_{1}\right)$. For a completely bounded linear map $\varphi: X \rightarrow Y$ between operator spaces $X$ and $Y$, we define the nonnegative real number $\gamma_{\mathrm{op}}(\varphi)$ and $\delta_{\mathrm{op}}(\varphi)$ by

$$
\begin{aligned}
& \gamma_{\text {op }}(\varphi)=\inf \left\{\gamma\left(\varphi_{n}\right): n=1,2, \ldots\right\} \\
& \delta_{\text {op }}(\varphi)=\inf \left\{\delta\left(\varphi_{n}\right): n=1,2, \ldots\right\}
\end{aligned}
$$

respectively.

Corollary 3.2. Let $\varphi: X \rightarrow Y$ be a completely bounded linear map between operator spaces $X$ and $Y$, with the dual map $\varphi^{*}: Y^{*} \rightarrow X^{*}$ between the operator duals $Y^{*}$ and $X^{*}$. Then we have the following:

(i) We have $\delta_{\mathrm{op}}(\varphi) \leq \gamma_{\mathrm{op}}\left(\varphi^{*}\right)$ : The equality $\delta_{\mathrm{op}}(\varphi)=\gamma_{\mathrm{op}}\left(\varphi^{*}\right)$ holds whenever $\varphi$ is surjective.

(ii) We have $\gamma_{\mathrm{op}}(\varphi) \geq \delta_{\mathrm{op}}\left(\varphi^{*}\right)$ : The equality $\gamma_{\mathrm{op}}(\varphi)=\delta_{\mathrm{op}}\left(\varphi^{*}\right)$ holds whenever $\varphi$ is injective.

Let $\varphi: X \rightarrow Y$ be a complete contraction, and so $\gamma_{\text {op }}(\varphi) \leq 1$ and $\delta_{\text {op }}(\varphi) \leq 1$. In this case, $\varphi$ is a complete isometry (respectively complete quotient) if and only if $\gamma_{\mathrm{op}}(\varphi)=1$ (respectively $\delta_{\mathrm{op}}(\varphi)=1$ ). Therefore, Corollary 3.2 may 
be considered as a generalization of a well-known theorem: $\varphi$ is a complete isometry if and only if $\varphi^{*}$ is a complete quotient; $\varphi$ is a complete quotient if and only if $\varphi^{*}$ is a complete isometry and $\varphi$ is surjective.

Corollary 3.3. Let $\varphi: X \rightarrow Y$ be a completely bounded linear map between operator spaces $X$ and $Y$. Then the following are equivalent:

(i) $\varphi$ is injective with $\gamma_{\mathrm{op}}(\varphi)>0$.

(ii) $\varphi^{*}$ is surjective with $\delta_{\mathrm{op}}\left(\varphi^{*}\right)>0$.

\section{References}

[1] C. Apostol, The reduced minimum modulus, Michigan Math. J. 32 (1985), 279-294.

[2] D. P. Blecher and C. Le Merdy, Operator Algebras and Their Modules, London Math. Soc. Monograph, N. S. Vol. 30, Oxford Sc. Publ., 2004.

[3] E. G. Effros and Z.-J. Ruan, Operator Spaces, London Math. Soc. Monograph, N. S. Vol. 23, Oxford Sc. Publ., 2000.

[4] E. G. Effros and C. Webster, Operator analogues of locally convex spaces, Operator Algebras and Applications (Samos, 1996), pp. 163-207, NATO Adv. Sci. Inst. Ser. C Math. Phys. Sci., 495, Kluwer Acad. Publ., Dordrecht, 1997.

[5] H. A. Gindler and A. E. Taylor, The minimum modulus of a linear operator and its use in spectral theory, Studia Math. 22 (1962), 15-41.

[6] S. Goldberg, Unbounded Linear Operators: Theory and Applications, McGraw-Hill, 1966.

[7] I. S. Hwang and W. Y. Lee, The reduced minimum modulus of operators, J. Math. Anal. Appl. 267 (2002), 679-694.

[8] T. Kato, Perturbation Theory for Linear Operators, Springer-Verlag, 1966.

[9] S.-H. Kye and Z.-J. Ruan, On the local lifting property for operator spaces, J. Funct. Anal. 168 (1999), 355-379.

[10] G. Pisier, Introduction to Operator Space Theory, London Math. Soc. Lecture. Note Ser. Vol. 294, Cambridge. Univ. Press, 2003.

[11] Z.-J. Ruan, Subspaces of $C^{*}$-algebras, J. Funct. Anal. 76 (1988), 217-230.

Department of Mathematics

Seoul National University

SEOUl 151-742, KoreA

E-mail address: kye@snu.ac.kr 